\title{
Meniscus Allograft Transplantation Obtained From Adult Patients Undergoing Total Knee Arthroplasty May be Used for Younger Patients After Lateral Discoid Meniscus Meniscectomy
}

\author{
Yuka Kimura, M.D., Yuji Yamamoto, M.D., Shizuka Sasaki, M.D., Eiji Sasaki, M.D., \\ Tomoyuki Sasaki, M.D., Eiichi Tsuda, M.D., and Yasuyuki Ishibashi, M.D.
}

\begin{abstract}
Purpose: To investigate the clinical outcomes following meniscus allograft transplantation (MAT) to replace removed discoid lateral meniscus using intact lateral meniscus obtained from patients undergoing total knee arthroplasty for medial compartment osteoarthritis. Methods: Pediatric patients who underwent MAT secondary to partial or total meniscectomy due to discoid lateral meniscus between August 2010 and November 2018 were identified. Lateral menisci with bone blocks were transplanted arthroscopically. The demographic data, range of motion, meniscal allograft failure, which was defined as removal of an implanted meniscus, and complications were recorded. The Lysholm score and the KellgrenLawrence (KL) grade on radiographs were evaluated preoperatively and at final follow-up. The Knee injury and Osteoarthritis Outcome Score was evaluated in the final follow-up. Results: Eight patients who were symptomatic after discoid lateral meniscectomy were included. Donated allografts were harvested from adult patients aged between 64 and 78 years (mean 70.5 years). The mean age at previous meniscectomy and MAT were 10.5 and 18.1 years, respectively. All patients were followed up for a mean time of 5.2 years, and there were no cases of meniscus failure and complication. The Lysholm score significantly improved from preoperative to the final follow-up. Preoperatively, 6 and 2 knees were of KL grades II and III, respectively. At final follow-up, no increased KL grade was observed in 5 patients; however, an increase by 1 grade was observed in 2 patients and an increase by 2 grades was observed in 1 patient. The Knee injury and Osteoarthritis Outcome Scores at the final follow-up were 87.2 for pain, 87.9 for symptoms, 95.4 for activities of daily living, 79.4 for sports, and 65.6 for quality of life. Conclusions: Older lateral meniscus allografts obtained from knees of adult patients undergoing total knee arthroplasty and transplanted into younger patients with defects from discoid lateral meniscectomy resulted in improved Lysholm scores but no improvement in Tegner scores or knee motion. KL grades were unchanged in 5 of the 8 patients at the final follow-up. Level of Evidence: IV, therapeutic case series.
\end{abstract}

From the Department of Orthopaedic Surgery (Y.K., Y.Y., S.S., E.S., Y.I.) and Department of Rehabilitation Medicine (E.T.), Hirosaki University Graduate School of Medicine; and Department of Orthopaedic Surgery, Hirosaki Memorial Hospital (T.S.), Hirosaki, Japan.

The authors report that they have no conflicts of interest in the authorship and publication of this article. Full ICMJE author disclosure forms are available for this article online, as supplementary material.

Investigation was performed at Hirosaki University Graduate School of Medicine, Hirosaki, Japan.

Received August 23, 2020; accepted July 13, 2021.

Address correspondence to Yuka Kimura, M.D., Department of Orthopaedic Surgery, Hirosaki University Graduate School of Medicine, Zaifu-cho 5, Hirosaki,0368562,Japan.E-mail:yukax10@hirosaki-u.ac.jp

(C) 2021 THE AUTHORS. Published by Elsevier Inc. on behalf of the Arthroscopy Association of North America. This is an open access article under the CC BY-NC-ND license (http://creativecommons.org/licenses/by-nc-nd/4.0/). 2666-061X/201458

https://doi.org/10.1016/j.asmr.2021.07.024
A previous study showed increased degenerative $\mathrm{A}_{\text {changes in the knees after meniscus removal or }}$ partial resection. ${ }^{1}$ Therefore, there has been an increased emphasis on the importance of meniscal repair in preventing osteoarthritis, ${ }^{2}$ and the currently it is recommended to repair meniscal tears as much as possible to preserve the meniscus. Patients with a symptomatic discoid lateral meniscus (DLM) with evidence of tears or instability usually require arthroscopic meniscectomy with or without peripheral repair. A DLM is susceptible to injuries associated with severe degeneration because of its known vulnerability; therefore, in some cases, total or subtotal meniscectomy has been performed, and this could cause a meniscal deficiency. ${ }^{3,4}$

In young patients, meniscal loss following subtotal or total meniscectomy can be devastating, leading to a loss 
in knee function, pain, and early onset of arthrosis. ${ }^{5}$ Advances in artificial meniscus implants and meniscal regeneration offer the promise of restoring lost meniscus function. However, any long-term preventive effect on the development of knee osteoarthritis remains controversial, and a surgical option has yet to be established. Furthermore, a tendon autograft was shown to be an unsuccessful substitute for an absent lateral meniscus in cases of valgus knees with severe degenerative lateral compartment arthritis. ${ }^{6}$

Meniscal allograft transplantation (MAT) is considered an effective treatment for pain relief and improved knee joint function after a previous subtotal or total meniscectomy in active and young patients. ${ }^{7-12}$ However, allograft tissues including menisci have limited availability in certain countries where human tissue banks are not yet established. ${ }^{13}$ Some patients who undergo total knee arthroplasty (TKA) for medial osteoarthritis have a good lateral meniscus, and this is usually removed and discarded. The purpose of this study was to investigate the clinical outcomes following MAT to replace removed DLM using intact lateral menisci obtained from patients who underwent TKA for medial compartment osteoarthritis. It was hypothesized that allograft meniscus obtained from patients who had TKA would be an available option in MAT.

\section{Methods}

The study design was approved by the ethics committee of the institution (\#2010-066), and all patients, as well as their parents (for patients younger the age of 20 years) signed an informed consent document. This retrospective cohort study was conducted between August 2010 and November 2018. Patients who underwent MAT following secondary to partial or total meniscectomy due to DLM were enrolled in this study.

The inclusion criteria for this study were as follows: patients with previous total or subtotal meniscectomy for DLM, symptomatic meniscal insufficiency after failure of conservative treatment, and a minimum 2- year follow-up duration. Patients were excluded if they had contralateral knee injury, osteoarthritis above KL4 or articular cartilage degeneration Internal Cartilage Repair Society grade IV, or combined ligament injuries, or a severe synovial reaction at the time of surgery. All patients were clinically assessed for ligamentous stability, and knee radiographs confirmed the closure of the proximal tibial and distal femur physis or revealed the drop-out sign on $\mathrm{T} 2$-gradient-recalled echo magnetic resonance imaging (MRI) ${ }^{14}$ before MAT.

Donated allografts were solicited from patients planned for TKA for the treatment of medial knee osteoarthritis who had no tear or degeneration in the lateral meniscus on routine preoperative MRI. The donors underwent a standard process in which a written consent was obtained and the procedure of donation was explained; in addition, donors were screened for transmissible infectious diseases, including hepatitis, serological test, and treponema pallidum antibody hemagglutination test for syphilis, human T-cell leukemia virus type 1 , and HIV. TKA was performed following a routine medial parapatellar approach. To minimize the invasion of the meniscal tissue, an electrical scalpel is not used, and the outer rim of meniscus and cruciate ligaments were sharply cut using a sharp-pointed knife. The tibial cut was made using an extramedullary guide with the anterior and posterior horns of the meniscus bound to the proximal part of the tibia. After irrigation with normal saline, the harvested menisci were placed in a sterilized secured plastic package. Afterwards, they were fresh frozen at $-80^{\circ} \mathrm{C}$ and not irradiated.

For sizing the graft, plain radiographs of both the donor and the recipient were obtained before surgery. Graft sizing was performed by measuring the anteroposterior width and the lateral length of the tibial plateau on plain radiographs. ${ }^{15}$ Mismatches within 3 $\mathrm{mm}$ were allowed. Frozen allografts were thawed in the saline solution containing polymyxin B using a sterile technique. The bone blocks were created measuring approximately 5 to $6 \mathrm{~mm}$ in diameter and 6 to 7 while maintaining the attachment of the anterior and posterior meniscal roots (Fig 1). Anterior, posterior, tibial,

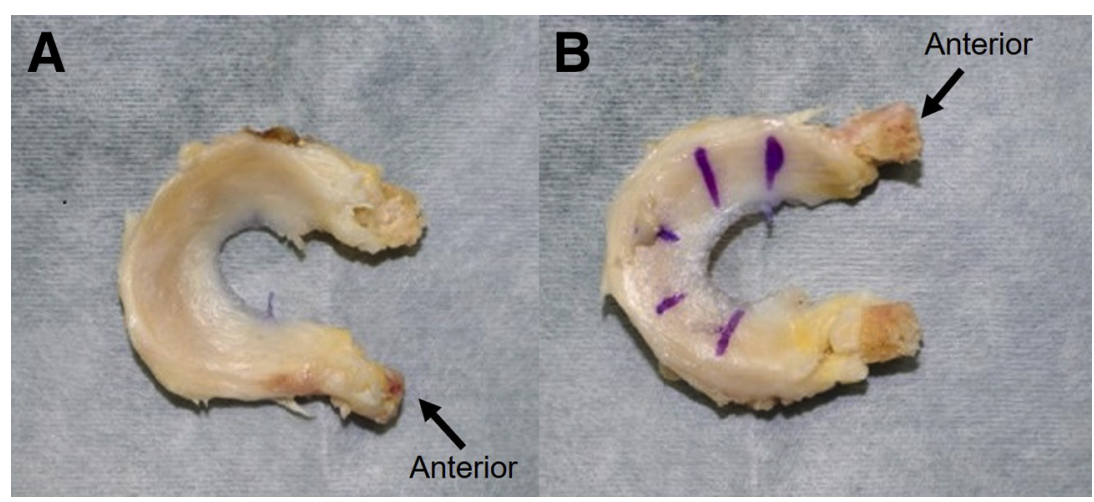

Fig 1. A prepared meniscus from the right knee with a bone block. (A) Femoral side, (B) tibia side. A blue line was created to identify the tibial side of the meniscus. The bone blocks were created measuring approximately 5 to $6 \mathrm{~mm}$ in diameter and 6 to $7 \mathrm{~mm}$ in lengths while maintaining the anterior (arrows) and posterior meniscal root attachment 
Fig 2. (A) Arthroscopic finding from lateral portal of the right knee. (B) Tibial guide pin for anterior and posterior foot prints of the lateral meniscus and graft insertion. The tibial guidewires (for the anterior and posterior anatomical footprints) were inserted under arthroscopic guidance, and then allograft meniscus was induced through the arthroscopic portal

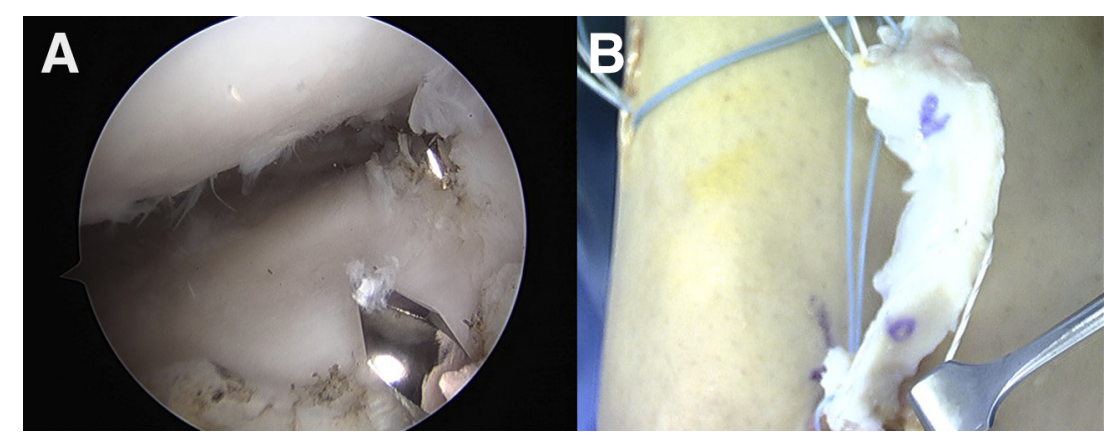

and femoral sides of the graft were identified by markings. The meniscus allograft was secured by sutures at the anterior horn, posterior horn, and mid-body to aid with insertion into the knee.

\section{Surgical Technique of MAT}

The senior author (Y.I.) simultaneously performed, or directly supervised (made all important surgical decisions) the MAT and all other treatments in these patients. Lateral menisci with bone blocks were transplanted arthroscopically. First, an arthroscopic examination was performed, and the meniscus and cartilage were assessed by probing. The remnant of the native meniscus was debrided up to the rim, and rasping was performed. The anatomical attachments of the posterior and anterior horns of the meniscus were identified; next, tibial guidewires (for the anterior and posterior footprints) were inserted with an anterior cruciate ligament tibial guide under arthroscopic guidance (Fig 2). These guidewires were overdilled to make sockets (approximately $15 \mathrm{~mm}$ in lengths) that corresponded to the measured diameter of the prepared grafts ( 5 or 6 $\mathrm{mm})$ at the anterior and posterior horns. Afterwards, bone tunnels were drilled using a $4.5-\mathrm{mm}$ cannulated drill to enable the passage of sutures. The passing stitches were inserted into each bone tunnels and passed to the lateral portal. In addition, the traction stitch (no. 2-0 ultra-high molecular weight polyethylene) was similarly passed from the medial side of the articular capsule to the lateral portal to enable easier control of the graft. The graft was inserted through the arthroscopic portal using a suture relay technique (Fig 2). The anterior and posterior horn stitches were passed down the tunnels, and the graft was manually tensioned. The donor meniscus was secured to the meniscal rim with a combination of inside-out sutures using a zone-specific cannula and all-inside anchorbased sutures. All-inside meniscus repair device was limited used in the posterior portion, and the cannulaguided ultra-high molecular weight polyethylene meniscus repair sutures were used for the anterior to posterior portion of the meniscus. Sutures were placed until the desired stability was achieved (Fig 3).
Different rehabilitation protocols were applied based on concomitant surgical procedures including osteotomy, or osteochondral autograft transplantation. Basically, in patients who underwent isolated MAT, a cylinder cast was applied postoperatively for 3 weeks and was not subjected to non-weight-bearing for 4 weeks. Partial weight-bearing was allowed for 2 to 4 weeks before full-weight bearing was allowed. Running was allowed starting between 3 and 6 months postoperatively, and return to sports activities was allowed after 6 to 9 months as appropriate.

Physical examinations were conducted on the day before surgery and at the final follow-up. Pre- and postoperative assessments included range of motion, Lysholm score, Tegner activity score, and the KellgrenLawrence (KL) grade on plain radiographs. The meniscal allograft failure, which was defined as removal of an implanted meniscus, complications, and any further surgery on the operated knee, were recorded. The Knee Injury and Osteoarthritis Outcome Score was used as an evaluation tool for patient-related outcome at the final follow-up. All patients routinely underwent postoperative MRI follow-up at 3, 6, and 12 months to evaluate the transplanted meniscus and cartilage. One

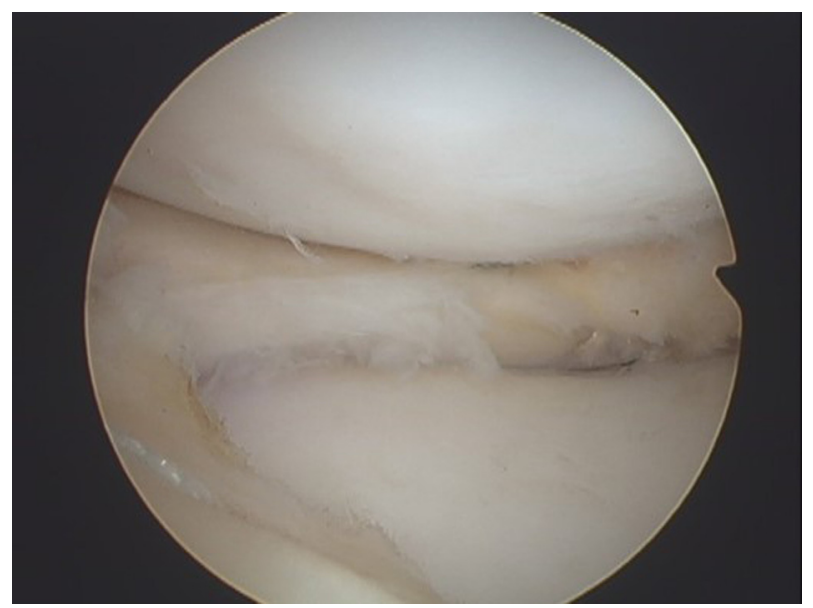

Fig 3. Arthroscopic finding from the lateral portal of the right knee after meniscus allograft transplantation. The donor meniscus was secured to the meniscal rim 


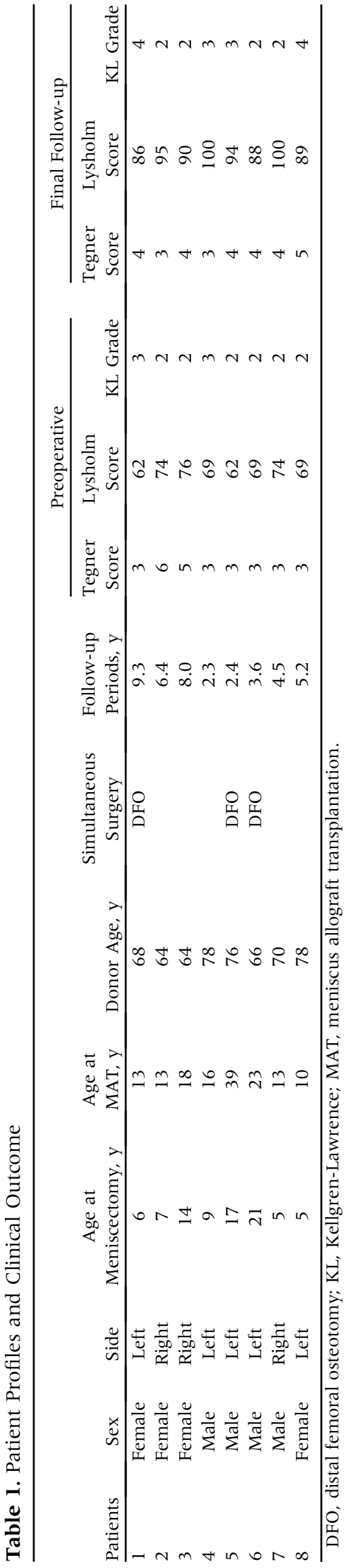

year after MAT, all patients underwent second-look arthroscopy to confirm the survival of the allograft. After 12 months, MRI was performed depending on knee symptoms.

\section{Statistical Analysis}

The paired $t$ test was used to compare the pre- and postoperative knee ranges of motion, Lysholm scores, and KL grades. The Wilcoxon signed-rank test was used to compare the pre- and postoperative Tegner activity scores. Notably, a significant level was set at $P<.05$. All statistical analyses were performed with the SPSS version 21 (IBM Corp., Armonk, NY).

\section{Results}

From August 2010 to November 2018, 8 symptomatic patients ( 4 female and 4 male) underwent MAT. The mean age at the time of previous meniscectomy and at the time of MAT were 10.5 (5-21) years and 18.1 (10-39) years, respectively. Of the 8 patients, MAT was conducted in the left and right knees for 5 and 3 patients, respectively. Of 8 patients, 3 simultaneously underwent distal femoral osteotomy.

At second-look arthroscopy l year after MAT, a stable peripheral healing and relatively good positioning of the allograft in relation to alignment of the tibiofemoral joint were observed in all cases. No meniscus retear, obvious shrinkage, or progression of articular cartilage degeneration were noted compared with the status at MAT.

All patients were followed-up for a mean postoperative period of 5.2 years (range, 2.3-9.3 years), and there were no cases of meniscus failure. The mean preoperative ranges of motion and the ranges of motion at the final follow-up were $142.5 \pm 13.6^{\circ}$, and $145.6 \pm$ $5.0^{\circ}$, respectively $(P=.460)$. The median Tegner activity scores were 3 (3-6) preoperatively, and 4 (3-5) at the final follow-up, respectively $(P=.334)$. The mean Lysholm score significantly improved from 69.4 points preoperatively to 92.8 points at the final follow-up $(P=.000)$. The Knee injury and Osteoarthritis Outcome Scores at the final follow-up were 87.2 points for pain, 87.9 points for symptoms, 95.4 points for activities of daily living, 79.4 points on sports/rec, and 65.6 points for quality of life. On plain radiographs, 6 knees demonstrated KL grade II and 2 knees demonstrated KL grade III changes preoperatively. At the final follow-up, no increase in KL grade osteoarthritis was observed in 5 patients. However, in 2 patients, osteoarthritis increased by one grade and in one patient it increased 2 grades. One patient underwent autologous chondrocyte implantation to the cartilage defect in the lateral femoral condyle at 43 months after MAT.

Patient details are shown in Table 1. Fig 4 shows a case of preoperative and postoperative radiographs and 


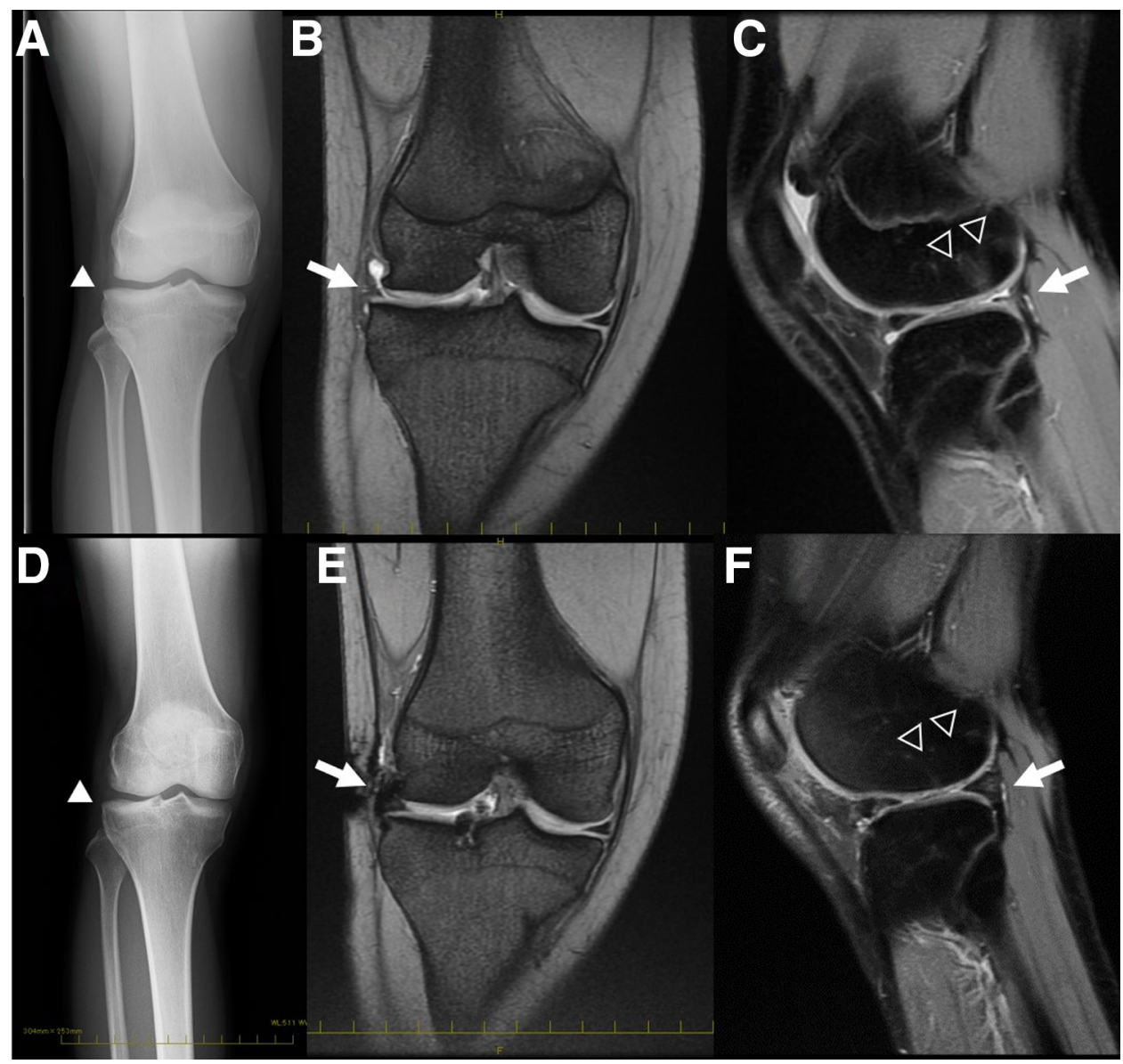

Fig 4. Pre- and postoperative findings in radiographs and magnetic resonance imaging (MRI) of the right knee in a 13 -year-old female patient. (A) Preoperative anteroposterior (AP) knee radiographs showed the definite osteophytes on the lateral tibial plateau and possible joint space narrowing of the lateral joint space (white arrowhead), diagnosed Kellgren-Lawrence grade II. (B-C) MRI before the meniscal allograft transplantation showed the absence of lateral meniscus (arrow) with bone marrow edema lesions (open arrow head) in the posterior aspect of the lateral femoral condyle and an irregular of articular cartilage. (D) Postoperative AP knee radiographs after 6 years' postoperatively. An increase in the degree of osteophyte on joint space narrowing (white arrowhead) was not observed. (E-F) MRI after 6 years' postoperatively showed in situ lateral meniscal allograft with a good hypointense signal on the coronal and sagittal plane (arrow), and healed bone edema at the lateral femoral condyle (open arrowhead).

MRI examinations findings of a the 13-year-old female patient (patient no. 2 of Table 1).

\section{Discussion}

In the cases in this study, the knees required MAT because of a partial or complete discoid lateral meniscectomy had been performed, and these patients were young. Furthermore, lateral meniscus allografts obtained from the knees of older patients (mean age 70.5 years) undergoing TKA were transplanted into younger patients (mean age 18.1 years), and these grafts achieved acceptable subjective and objective clinical outcome at the final follow-up. Arthroplasty is not indicated young patient with early-stage osteoarthritis, and surgical treatment using other artificial meniscus implants or autograft tissue has not proven to be useful on the long-term. Because there are no alternative treatments, MAT was the last resort in the treatment of young symptomatic patients with meniscal deficiency. However, allograft meniscus tissues have limited availability in some countries that are yet to establish systems for tissue banking. Therefore, the allograft menisci used were donated by patients who underwent TKA.

The lateral meniscus contributes to load-bearing more than the medial meniscus. ${ }^{16}$ The increased loading of the femorotibial compartment with weight bearing in a knee with an absent meniscus has been demonstrated to be detrimental to the underlying articular cartilage. ${ }^{17}$ Total and subtotal meniscectomy were previously preferred in the treatment of a torn DLM, and the clinical outcomes with short- to mid-term follow-up 
after surgery shows good results. ${ }^{5}$ However, long-term outcomes of these procedures revealed a high incidence of osteoarthritis. ${ }^{4,18,19}$ DLM should be treated minimal meniscectomy with repair to restore the function of the meniscus. ${ }^{20}$

MAT has been performed in the past decades for the treatment of meniscectomized knees; however, there have been few reports on MAT after meniscectomy of a DLM. Kim et al. ${ }^{21}$ first reported MAT in 14 patients who previously underwent total or subtotal resection of the DLM, and showed good results in terms of improvement in clinical symptoms, allograft survival, and prevention of degenerative arthritis after a mean follow-up duration of 58.1 months. Yoon et al. ${ }^{22}$ compared the clinical results of MAT after total meniscectomy in 16 patients of DLM and 20 with nonDLM and reported that the objective knee function, subjective satisfaction, and extrusion of the graft on MRI were not significantly different between the discoid and non-discoid groups after a minimal 2-year follow-up. Zaffagnini et al. ${ }^{4}$ reported a 15 -year-old woman with functional limitation who underwent MAT with femoral osteotomy following total meniscectomy for DLM. After 15-years of follow-up, good clinical and MRI-related outcomes were achieved. Although no strong evidence is available on MAT chondroprotection after long-term follow-up, MAT is considered as an effective method for reducing the pain caused by meniscal deficiency and improving knee joint function.

The biomechanical properties of lateral meniscal allografts obtained from patients undergoing TKA with isolated medial femorotibial osteoarthritis could be similar to that of frozen specimens. ${ }^{23}$ However, the MAT outcomes using aged meniscus allografts are yet to be conclusively determined. The donors in this study were patients that were $70.5 \pm 6.0$ years of age, and were older than those in previous studies (45-63.8 years). ${ }^{23,24}$ Bursac et al. ${ }^{24}$ examined the tensile property and biomechanical composition including collagen, proteoglycan, and water content of lateral and medial menisci ranging from 15 to 44 years of age harvested and immediately stored at $-80^{\circ} \mathrm{C}$. They concluded that there were no significant correlations between the biomechanical composition or tensile mechanical properties and donor age of lateral or medial menisci.

In this clinical study, the knees required the MAT because a partial or complete discoid lateral meniscectomy had been performed and these were younger patients. Although age-dependent alternation in the biomechanical or biochemical property of a meniscus allograft could potentially affect its long-term function, ${ }^{25}$ the patients in this study had improved Lysholm scores and good subjective scores at the final follow-up, and these scores were comparable with the outcomes after MAT in previous studies on adolescents and younger individuals. ${ }^{26,27}$ However, 5 of 8 patients maintained the KL grade after a mean follow-up duration of 5.2-years. Further long-term follow-up is needed for as long as least 20 to 30 years in young children.

\section{Limitations}

This study has a number of limitations. It was retrospective, limited to 8 patients, not randomized, without a control group, and had short- to mid-term follow-up.

\section{Conclusions}

Lateral meniscus allografts obtained from knees of adult patients undergoing TKA and transplanted into younger patients with defects from previous discoid lateral meniscectomy resulted in improved Lysholm scores but no improvement in Tegner scores or knee motion. KL grades were unchanged in 5 of the 8 patients at the final follow-up.

\section{References}

1. Petty CA, Lubowitz JH. Does arthroscopic partial meniscectomy result in knee osteoarthritis? A systematic review with a minimum of 8 years' follow up. Arthroscopy 2011;27:419-424.

2. Stein T, Mehling AP, Welsch F, von Eisenhart-Rothe R, Jager A. Long-term outcome after arthroscopic meniscal repair versus arthroscopic partial meniscectomy for traumatic meniscal tears. Am J Sports Med 2010;38:1542-1548.

3. Bin SI, Kim JC, Kim JM, Park SS, Han YK. Correlation between type of discoid lateral menisci and tear pattern. Knee Surg Sports Traumatol Arthrosc 2002;10:218-222.

4. Zaffagnini S, Espinosa M, Neri MP, Marcacci M, Grassi A. The treatment of meniscal deficiency with meniscal allograft transplantation and femoral osteotomy in a patient with history of lateral discoid meniscus. JBJS Case Connect 2020;10:e0079.

5. Lee YS, Teo SH, Ahn JH, Lee OS, Lee SH, Lee JH. Systematic review of the long-term surgical outcomes of discoid lateral meniscus. Arthroscopy 2017;33:1884-1895.

6. Johnson LL, Feagin JA Jr. Autogenous tendon graft substitution for absent knee joint meniscus: A pilot study. Arthroscopy 2000;16:191-196.

7. Bloch B, Asplin L, Smith N, Thompson P, Spalding T. Higher survivorship following meniscal allograft transplantation in less worn knees justifies earlier referral for symptomatic patients: Experience from 240 patients. Knee Surg Sports Traumatol Arthrosc 2019;27:1891-1899.

8. Elattar M, Dhollander A, Verdonk R, Almqvist KF, Verdonk P. Twenty-six years of meniscal allograft transplantation is it still experimental? A meta-analysis of 44 trials. Knee Surg Sports Traumatol Arthrosc 2011;19:147-157.

9. Grassi A, Bailey JR, Filardo G, Samuelsson K, Zaffagnini S, Amendola A. Return to sport activity after meniscal allograft transplantation: At what level and at what cost? A systematic review and meta-analysis. Sports Health 2019;1 1: 123-133.

10. Middleton S, Asplin L, Stevenson C, Thompson P, Spalding T. Meniscal allograft transplantation in the 
paediatric population: Early referral is justified. Knee Surg Sports Traumatol Arthrosc 2019;27:1908-1913.

11. Samitier G, Alentorn-Geli E, Taylor DC, et al. Meniscal allograft transplantation. Part 2: Systematic review of transplant timing, outcomes, return to competition, associated procedures, and prevention of osteoarthritis. Knee Surg Sports Traumatol Arthrosc 2015;23:323-333.

12. Verdonk PC, Verstraete KL, Almqvist KF, et al. Meniscal allograft transplantation: long-term clinical results with radiological and magnetic resonance imaging correlations. Knee Surg Sports Traumatol Arthrosc 2006;14:694-706.

13. Uchio Y. Meniscal allograft transplantation, still unaccepted treatment option in Japan? A review. Jpn J Med 2018;1:175-182.

14. Sasaki T, Ishibashi Y, Okamura Y, Toh S, Sasaki T. MRI evaluation of growth plate closure rate and pattern in the normal knee joint. J Knee Surg 2002;15:72-76.

15. Pollard ME, Kang Q, Berg EE. Radiographic sizing for meniscal transplantation. Arthroscopy 1995;1 1:684-687.

16. Gilbert S, Chen T, Hutchinson ID, et al. Dynamic contact mechanics on the tibial plateau of the human knee during activities of daily living. J Biomech 2013;16:555-551.

17. Berg B, Roos EM, Englund M, et al. Development of osteoarthritis in patients with degenerative meniscal tears treated with exercise therapy or surgery. A randomized controlled trial. Osteoarthritis Cartilage 2020;28:897-906.

18. Ahn JH, Kim KI, Wang JH, Jeon JW, Cho YC, Lee SH. Long-term results of arthroscopic reshaping for symptomatic discoid lateral meniscus in children. Arthroscopy 2015;31:867-873.

19. Okazaki K, Miura H, Matsuda S, Hashizume M, Iwamoto Y. Arthroscopic resection of the discoid lateral meniscus: long-term follow-up for 16 years. Arthroscopy 2006;22:967-971.

20. Yamasaki S, Hashimoto Y, Takigami J, Terai S, Takahashi S, Nakamura H. Risk factors associated with knee joint degeneration after arthroscopic reshaping for juvenile discoid lateral meniscus. Am J Sports Med 2017;45: 570-577.

21. Kim JM, Bin SI. Meniscal allograft transplantation after total meniscectomy of torn discoid lateral meniscus. Arthroscopy 2006;22:1344-1350.el.

22. Yoon KH, Lee SH, Park SY, Jung GY, Chung KY. Meniscus allograft transplantation for discoid lateral meniscus: Clinical comparison between discoid lateral meniscus and nondiscoid lateral meniscus. Arthroscopy 2014;30:724-730.

23. Jacquet C, Erivan R, Sharma A, et al. Preservation methods influence the biomechanical properties of human lateral menisci: An ex vivo comparative study of 3 methods. Orthop J Sports Med 2019;29:7 2325967119841622.

24. Bursac P, York A, Kuznia P, Brown LA, Arnoczky SP. Influence of donor age on the biomechanical and biochemical properties of human meniscal allografts. Am J Sports Med 2009;37:884-889.

25. Lubowitz JH, Verdonk PCM, Reid JB, Verdonk R. Meniscus allograft transplantation: A current concepts review. Knee Surg Sports Traumatol Arthrosc 2007;15:476-492.

26. Riboh JC, Tilton AK, Cvetanovich GL, Campbell KA, Cole BJ. Meniscal allograft transplantation in the adolescent population. Arthroscopy 2016;32:1133-1140.el.

27. Novaretti JV, Patel NK, Lian J, et al. Long-term survival analysis and outcomes of meniscal allograft transplantation with minimum 10-year follow-up: A systematic review. Arthroscopy 2019;35:659-667. 